\title{
From Business Process Model to Application System - Developing an Information System with the House of Business Engineering (HOBE)
}

\author{
August-Wilhelm Scheer, Michael Hoffmann \\ Institut für Wirtschaftsinformatik (IWi) an der Universität des Saarlandes \\ Im Stadtwald, Geb. 14.1, 66123 Saarbrücken \\ \{scheer, hoffmann\}@iwi.uni-sb.de
}

\begin{abstract}
Organizational concepts, like virtual enterprises or profit centers in companies, are ordering new functionality on information systems. Enterprises have to customize their business processes in very short periods because of stronger competition and globalization. Concepts like electronic commerce and supply chain management need a permanent optimization of the business processes along the supply chain. The generating of individual software and model based customizing of ERP-packages offer potentials for more flexibility supporting business processes with information systems. This paper shows a concept for describing and implementing business processes and a software development project using this concept.
\end{abstract}

\section{Flexible Information Systems for Mobile Enterprises}

Organizational concepts, like virtual enterprises or profit centers in companies, are ordering new functionality on information systems. The networks between many organizational units in a company and between companies need more flexibility in implementing and customizing information systems.

In the past, information systems had been customized on the basis of organizational structures and business processes once and then not been altered again over a period of many years. Nowadays, enterprises have to customize the business processes in very short periods because of stronger competition and globalization. Concepts like electronic commerce and supply chain management demand a permanent optimization of the business processes along the supply chain.

Companies selling enterprise resource planning packages have realized the necessity to deliver tools reducing the time and the costs spent in implementing and customizing their software products.

Many enterprises expect more flexibility from generating their individual software products on the basis of semantic models and software components working together in a framework. A change of the semantic models automatically takes effect on the configuration of the information system.

With ARIS - House of Business Engineering, chapter 2 introduces a concept for describing business processes from semantic models to implementation. Chapter 3 shows the development of software applications using the HOBE-concept. In chapter 4 future trends are presented. 


\section{ARIS - House of Business Engineering (HOBE)}

ARIS - House of Business Engineering is an integrated concept describing and executing business processes. Furthermore, it is a framework for developing real application software. The next chapters explain the meaning of the different levels shown in Fig. 1.

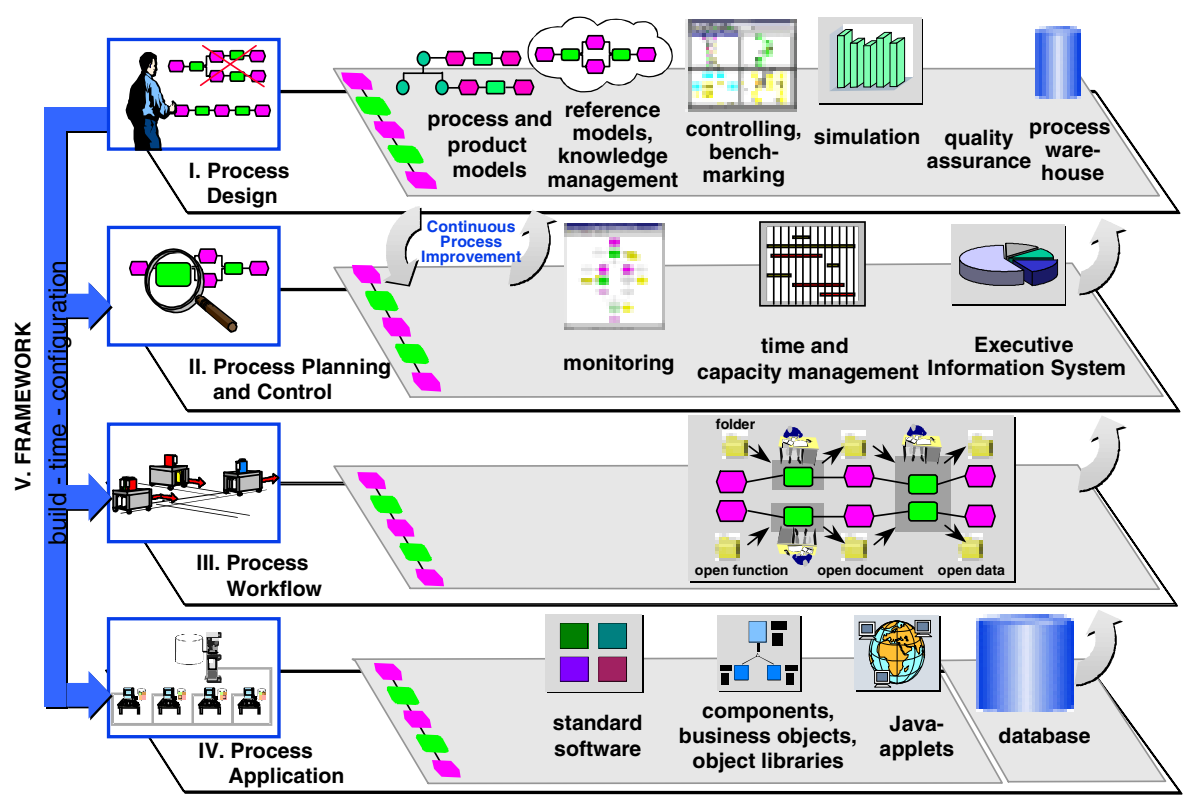

Fig. 1 ARIS - House of Business Engineering

\subsection{Level I: Process Design}

Level I describes business processes according to the routing. Therefore, the ARIS concept provides a method to cover every aspect of the business process. Methods for optimizing and guaranteeing the quality of processes are available, too.

\subsection{Level II: Process Management}

Level II plans and monitors every current business process from the "business process owner's" point of view. Various methods of scheduling and capacity control and cost analysis are available. By monitoring the process, the process manager is aware of the status of each process instance. 


\subsection{Level III: Process Workflow}

Level III transports the objects to be processed, such as customer orders with the corresponding documents or insurance claims in insurance companies, from one workplace to the next. The documents are then stored in "folders". Workflow systems carry out the material handling in electronically stored documents.

\subsection{Level IV: Process Application}

Level IV processes the documents transported to the individual workplaces; that is where the functions of the business process are executed. At this level, computeraided application systems - from simple word processing programs to complex standard software modules and internet applets - are used.

\subsection{Combination of the Four Levels and Framework}

Those four levels of ARIS - House of Business Engineering are connected interdependently. Level II (Process Management) delivers information about the profitability of currents processes. That information forms the basis for a continuous adjustment and improvement of the business processes at Level I. The process workflow reports data regarding times, amounts and organizational allocation etc. to Process Management. The workflow systems at Level III start applications at Level IV.

The framework is the fifth component, which includes all four levels. It combines architecture and application knowledge, from which concrete applications are configured. At all levels of the HOBE concept, the ARIS life cycle model can be used. That means that the software can be described by Requirements Definition, Design Specification and Implementation.

The following chapter describes the prototypical implementation of the HOBE Concept.

\section{Prototypical Implementation of ARIS - House of Business Engineering}

In order to implement the HOBE Concept, the institute for information systems (Institut für Wirtschaftsinformatik - IWi) of the University of Saarland, and the IDS Scheer AG, have launched a project to prototypically implement a sales scenario. This project was supported by CSE Systems Cooperation and NEXUS GmbH. Fig. 2 shows the components used with the implementation. 


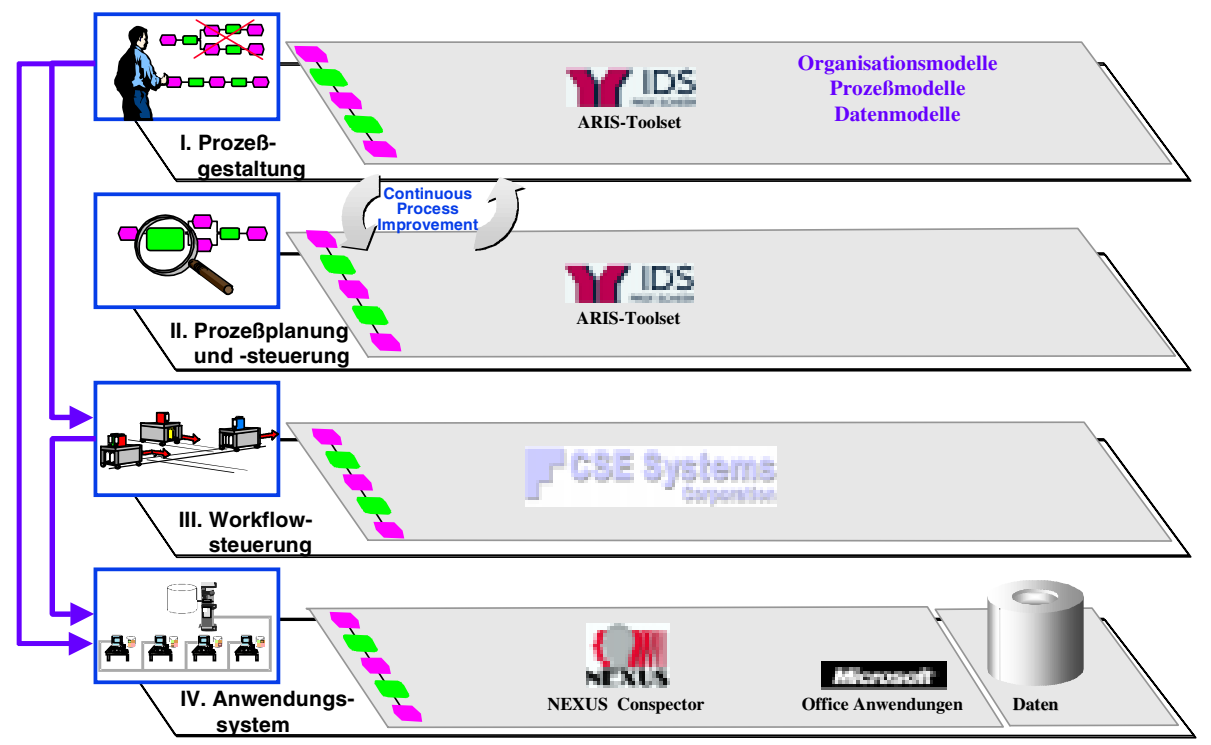

Fig. 2 Components used with the implementation

At Level I, the ARIS Toolset has been used to model the processes, the data structure and the hierarchical organization of the enterprise considered. An organigram has been modeled for the hierarchical organization, as well as an UML class diagram for the data structure and an extended Event Driven Process Chain (eEPC) for the process organization.

\subsection{Data Modeling}

On the basis of the UML class diagram, a database repository has been generated by using a report and an interface between the ARIS Toolset and the NEXUS Builder.

The following rules were observed:

- Each class is represented as an object in the database.

- Each association with the cardinality m:n is represented as an object in the database.

- Associations with the cardinality 1:n are not represented as objects. The attributes of these associations are administered in the subordinate classes.

- Classes are depicted centrally in forms, associated classes are depicted in the detail section.

Filling the generated database with content, one gets the company-wide integrated database/data set for the company considered. 


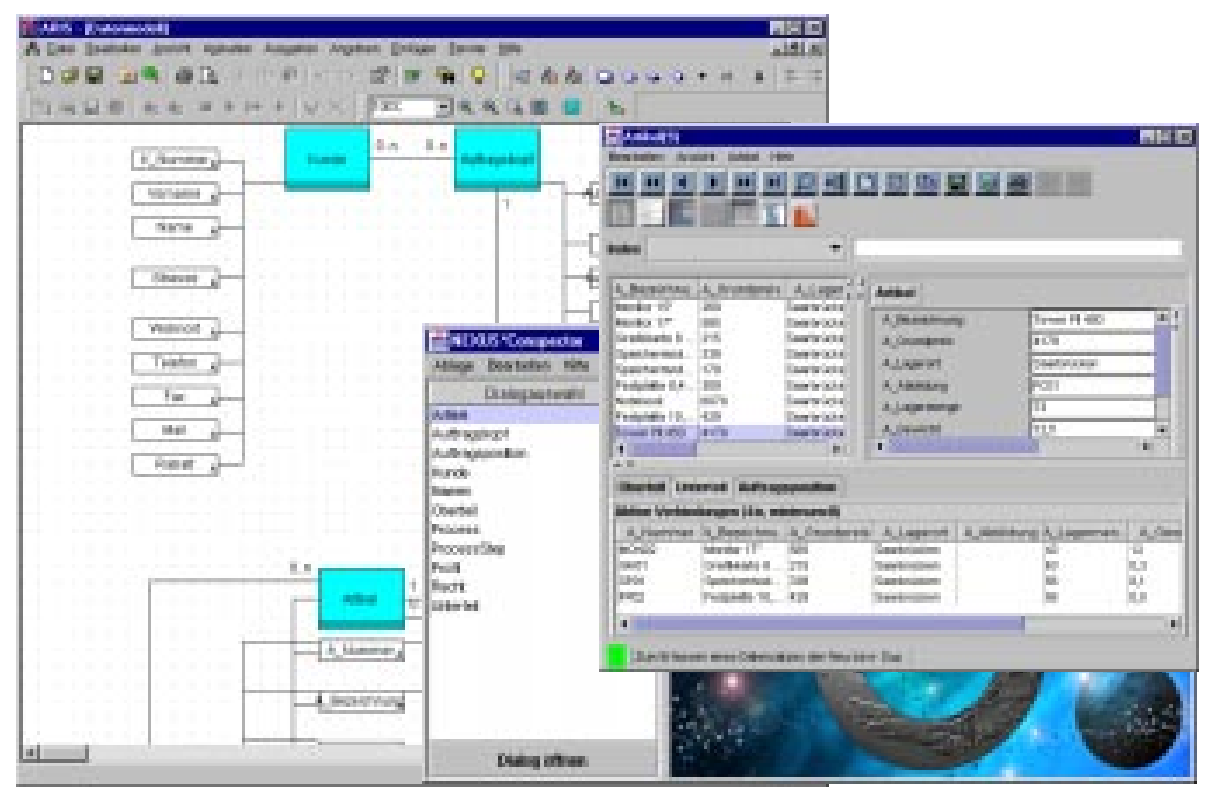

Fig. 3 Generating the Database

Fig. 3 shows part of the data model and the result after the transition to the NEXUS runtime environment. In the center of the generated mask, the class "Artikel" can be seen. In the detail section, the associations "Unterteil" and "Oberteil" can be faded in, which depict the bill of material view respectively. If someone selects the association "Auftragsposition", it will be depicted which order position an item has been assigned to.

\subsection{Organizational Structure}

Figure 4 shows the organigram of the exemplary company. As shown on the righthand side of the figure, in CSE-Workflow, the modeled organizational units are depicted as tree-control. The following transition rules have been used:

- An organizational unit in the organigram becomes an organizational unit in CSEWorkflow.

- An internal person is depicted as a user in CSE-Workflow.

- A person type in the organigram determines a role in CSE-Workflow.

- A position describes a synonym. 


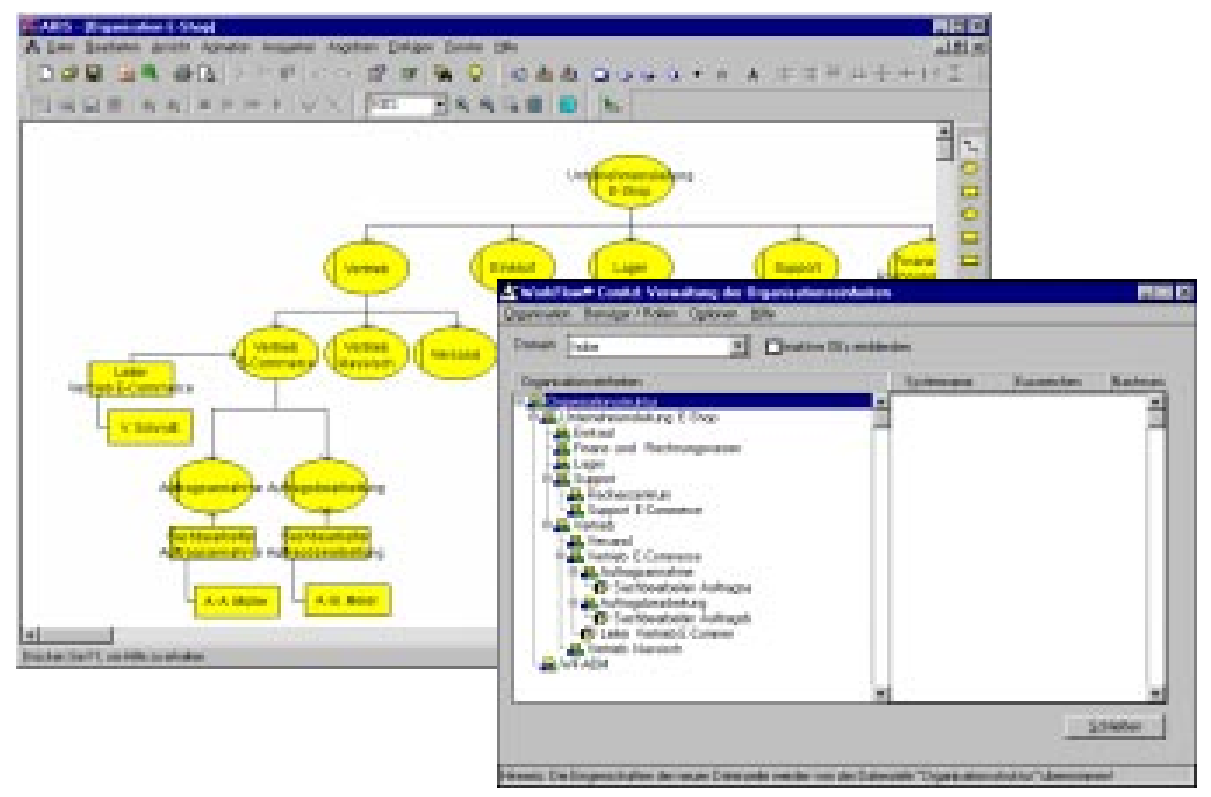

Fig. 4 Generating the Organizational Structure

\subsection{Organization of Workflow}

Fig. 5 shows the modeling of the workflow organization according to the modeling conventions of the CSE-Workflow filter, and the transition of the eEPC to CSEWorkflow. In the process designer of the CSE-workflow system, events are not depicted. The red-colored ovals stand for activities and correspond to the functions within the eEPC. The first activity is particularly stressed and may not be automated in the workflow.

It is unusual that application systems supporting the execution of a function are not modeled to the respective function, but to the event triggering the function. One reason for this is that events in workflow systems are not modeled and that application systems are opened via internal workflow triggers at the transitions. In Fig. 5 they are shown as exclamation marks on the lines between the activities. The organizational units are assigned to the functions responsible for their execution. 


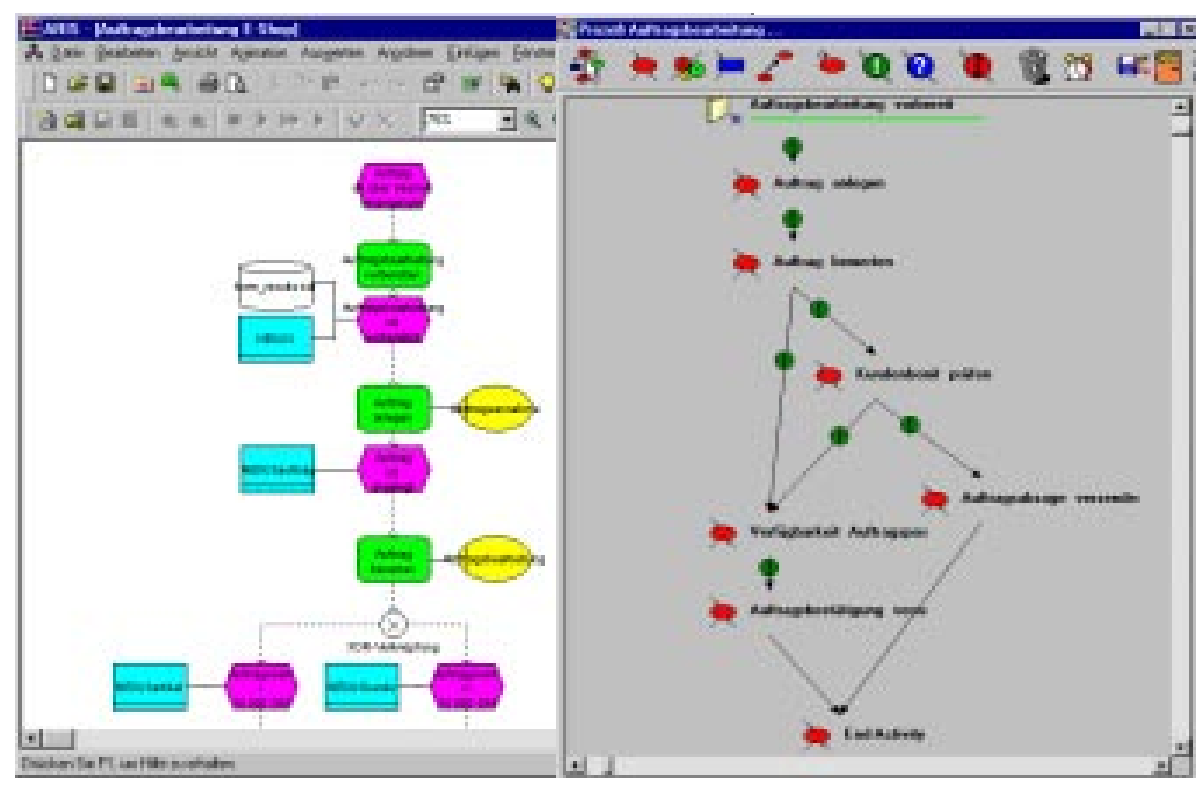

Fig. 5 Generating the Organization of Workflow

Next chapter describes the scenario supported with the developed information system.

\subsection{Scenario}

We consider an enterprise that sells computers and computer parts on the Internet. A customer can enter his master data in a form on the homepage of the enterprise and generate an order by entering quantities of the desired items. The generated electronic order automatically initiates the instantiation of a workflow. The employee responsible for order acceptance finds the form completed by the customer in the inbox and copies the data down to the NEXUS database generated from the ARIS toolset. When double-clicking on that business event, the database is automatically opened with the respective input mask appearing. Having finished his task, the employee sends the work folder containing the order to the next position according to the workflow model. The information systems required for the processing of the task are, too, opened by double-clicking on the business event. The business event will be forwarded to the next position until it has been worked off. Figure 6 shows the process model modeled with ARIS toolset, the Internet form which - when completed - triggers a workflow, the inbox of the employee responsible for order acceptance and the input mask of the generated enterprise-wide database. 


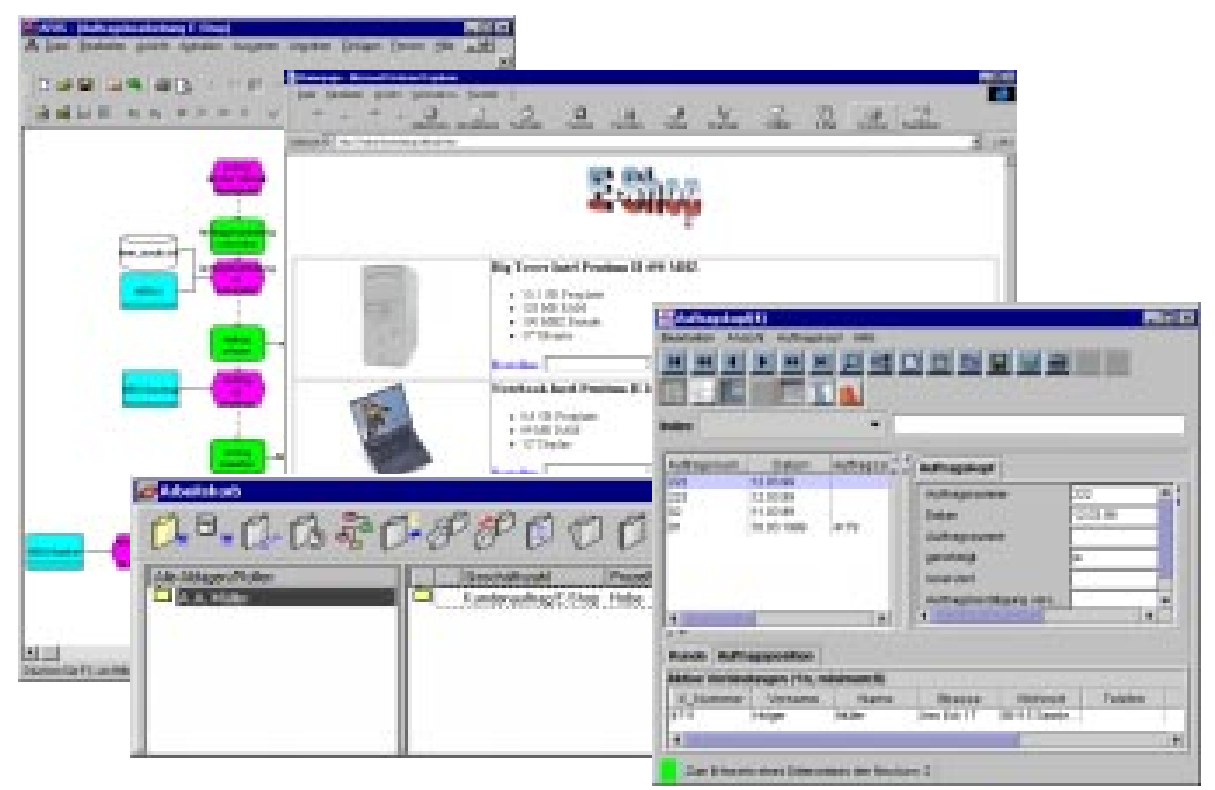

Fig. 6 Order processing with the system developed

\section{Outlook}

The development of the project shows that future models will not only document software, but also generate and modify it. Thus the importance of a consistent and complete modeling of enterprises on the level of requirements definition is growing. As a change of models automatically generates a change in the underlying software. Enterprise are able to support new business processes with application software or adjust existing business processes nearly without any delay. Thus ,e.g., the instantiation of ad-hoc workflow becomes feasible. New aspects are expected with reverse engineering and reverse customizing. The features of the tool used for modeling are very important. Besides usability, the functionality of the database that records the objects of the models, and the interfaces offered to application software programming environments are decisive for the exhaustion of maximum potentials. 